\title{
Jaundice with hypertrophic pyloric stenosis as an early manifestation of Gilbert syndrome
} Pascale Trioche, Jacqueline Chalas, Jeanne Francoual, Liliane Capel, Albert Lindenbaum,
Michel Odièvre, Philippe Labrune

\begin{abstract}
Jaundice associated with hypertrophic pyloric stenosis was recognised in three patients; previous reports have suggested that this is a possible early manifestation of Gilbert syndrome. Most patients with Gilbert syndrome are homozygous for a (TA) 7 TAA polymorphism in the gene promoter coding for bilirubin glucuronosyltransferase. Two of the reported patients were homozygous for the (TA) 7 TAA polymorphism whereas the third was heterozygous for the same polymorphism. Furthermore, no other factors contributing to jaundice in the three patients were found. These results suggest that jaundice associated with hypertrophic pyloric stenosis is due to molecular defects within the gene promoter.

(Arch Dis Child 1999;81:301-303)
\end{abstract}

Keywords: jaundice; pyloric stenosis; Gilbert syndrome

Jaundice is sometimes observed in infants with hypertrophic pyloric stenosis. ${ }^{1}$ Previous reports favoured the hypothesis that most cases of jaundice associated with pyloric stenosis could represent an early manifestation of Gilbert syndrome. ${ }^{2}$ Moreover, the low activity of bilirubin glucuronosyltransferase (BGT) observed in two patients, 4-5 months after they had been operated on for pyloric stenosis while jaundiced, suggested a permanent deficiency in bilirubin glucuronidation. ${ }^{3}$

Recent molecular studies of patients with Gilbert syndrome have shown the presence of a homozygous dinucleotide insertion of the TATA box in most cases ${ }^{4}$; conversely, few patients bore either heterozygous missense mutations, ${ }^{6}$ or rarely, homozygous missense mutations $^{7}$ in the coding sequence of the gene encoding BGT.

We report three patients who had jaundice associated with hypertrophic pyloric stenosis. Two of them had a homozygous TA dinucleotide insertion in the TATA box of the BGT gene, and the third one was heterozygous for the same TA dinucleotide insertion (whereas the 5 exons and the exon-intron junctions were normal). These results suggest that jaundice associated with pyloric stenosis is due to molecular defects within the TATA box of the BGT gene and suggest that this condition is an early manifestation of Gilbert syndrome.

\section{Case reports}

CASE 1

This $3500 \mathrm{~g}$ male infant was born after a full term uneventful pregnancy. His parents were healthy and unrelated and had no history of icteric episodes. He was bottle fed and not jaundiced when he was discharged. At 3 weeks old he was admitted to hospital because of projectile vomiting. He then weighed $4100 \mathrm{~g}$ and was mildly icteric. Serum bilirubin was $135 \mu \mathrm{mol} / 1$, entirely unconjugated. Pyloric stenosis was diagnosed and pyloromyotomy performed. The jaundice cleared within two days and the infant was discharged.

\section{CASE 2}

This $3300 \mathrm{~g}$ male infant was born after a full term uneventful pregnancy. His parents were healthy and unrelated and had no history of icteric episodes. He was bottle fed and jaundice did not develop during the first 10 days. At 4 weeks old he was admitted to hospital because of projectile vomiting. He then weighed $3950 \mathrm{~g}$ and was mildly icteric. Serum bilirubin was $165 \mu \mathrm{mol} / 1$, entirely unconjugated. Pyloric stenosis was diagnosed and pyloromyotomy was performed. The jaundice cleared within three days and the infant was discharged.

CASE 3

This 30 year old woman presented with mild jaundice. She had been operated on for pyloric stenosis at the age of 4 weeks and jaundice was noted at that time. She had been bottle fed until weaning, and had several icteric episodes since the age of 6 years, often triggered by infections. Except for mild jaundice, physical examination was normal. Laboratory data showed isolated unconjugated hyperbilirubinaemia $(80 \mu \mathrm{mol} / \mathrm{l})$.

\section{Methods}

Ten infants with hypertrophic pyloric stenosis but without jaundice were studied as controls. Genomic DNA was extracted from peripheral leukocytes. In the three patients, the 5 exons (including the exon-intron junctions and the splice-donor sites) and the promoter (including the TATA box) of the BGT gene were PCR (polymerase chain reaction) amplified; primers and experimental conditions have been reported previously. ${ }^{4}$ The amplified DNA fragments were then directly sequenced. In controls, only the promoter of the BGT gene was PCR amplified and sequenced.

\section{Results}

Patients 1 and 3 were homozygous for (TA) ${ }_{7}$ TAA, whereas patient 2 was heterozygous for (TA) 7 TAA (fig 1). In the three patients, the entire coding sequence, including the exonintron junctions and the splice donor sites, was normal. The 10 control infants were homozygous for the (TA) 6 TAA sequence (fig 1). 


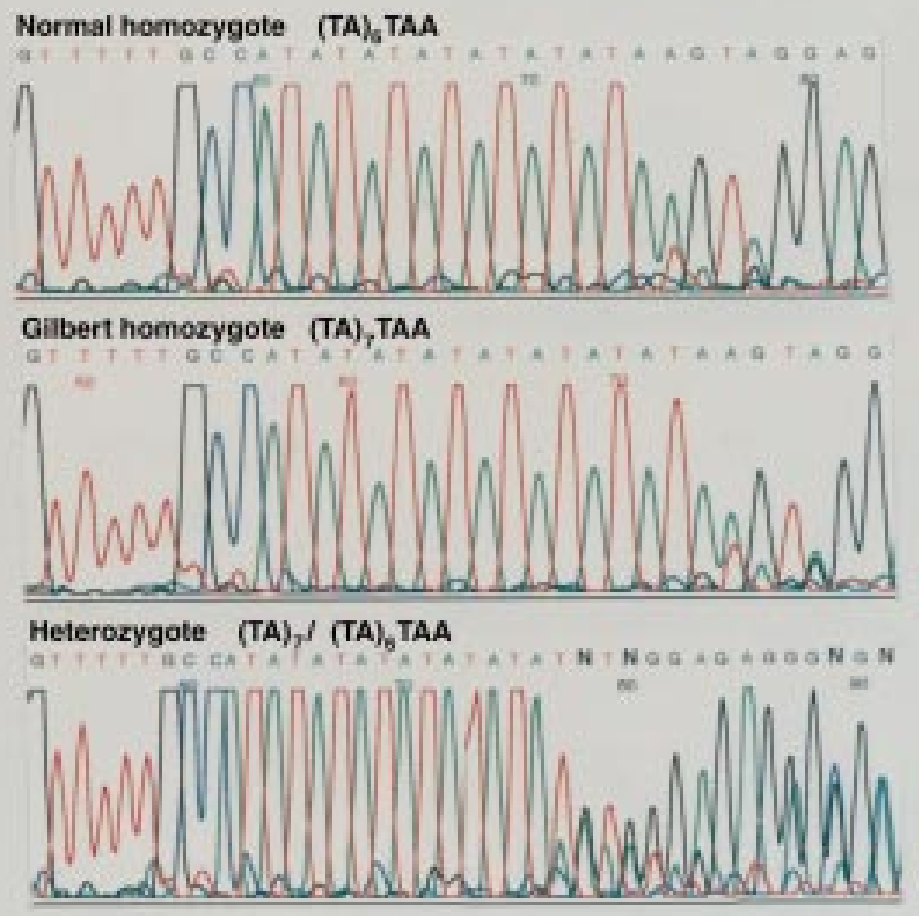

Figure 1 Nucleotide sequence of the BGT gene promoter. The first lane shows the normal sequence of the TATA box $\left(T A_{6} / T A_{6}\right)$. The second shows the homozygote Gilbert sequence of the TATA box $\left(T A_{7} / T A_{7}\right)$. The third shows the heterozygous Gilbert sequence of the TATA box $\left(T A_{6} / T A_{7}\right)$.
Gilbert syndrome was confirmed as this polymorphism is found in most patients with this condition. The third infant was heterozygous for the (TA) ${ }_{7}$ TAA polymorphism and a heterozygous missense mutation was thus carefully sought. The 5 exons (including the exon-intron junctions) were sequenced three times, following three independent PCR reactions, and no base substitution was identified. It is possible we missed a nucleotide substitution within an intron, however, such findings have never been observed in either Gilbert or Crigler-Najjar syndromes. ${ }^{14}$ Thus, it is likely that the heterozygous (TA) TAA polymorphism is the only nucleotide variant in the BGT gene in this infant.

Bosma and colleagues ${ }^{4}$ suggested that (TA) ${ }_{7}$ TAA homozygosity was necessary but not sufficient for the development of clinically apparent Gilbert syndrome; they speculated that additional factors disturbing the metabolism of bilirubin were necessary (increase in bilirubin load, defective hepatic uptake); however, this report concerned adult patients. Bancroft and colleagues ${ }^{10}$ have shown that even though peak jaundice levels did not differ among groups, neonates with homozygous (TA) $)_{7}$ TAA had an accelerated increase in jaundice during the first 2 days of life, whereas neonates with heterozygous (TA) 7 TAA were not significantly different in this respect from (TA) ${ }_{6}$ TAA homozygotes. However, this report did not consider the consequences of the polymorphism on the duration of jaundice in the neonatal period. In our three patients, other factors contributing to jaundice were carefully sought but were not found: all of them were full term, bottle fed infants with no clinical or biological evidence of infection, haemolysis (blood groups and Coombs tests) or hypothyroidism. Thus, these findings suggest that the promoter polymorphism was the only factor likely to contribute to jaundice in these three patients; this polymorphism has been showed to reduce the expression of the BGT gene by in vitro expression studies ${ }^{4}$ and fasting (induced by hypertrophic pyloric stenosis) is contributing to the increase of bilirubin production.

Our findings indicate an association between the promoter polymorphism of the BGT gene and jaundice associated with hypertrophic pyloric stenosis. It appears that this condition may be due to either homozygous or heterozygous (TA) ${ }_{7}$ TAA polymorphism.

have been identified. ${ }^{67}$ Moreover, recent studies have indicated a racial variability in the gene promoter. ${ }^{9}$ Other studies have shown that Gilbert syndrome is likely to accelerate the development of neonatal jaundice, ${ }^{10}$ to contribute to prolonged neonatal unconjugated hyperbilirubinaemia, ${ }^{11}$ and to interact with hereditary haemolysis, such as glucose- 6 phosphate dehydrogenase deficiency ${ }^{12}$ or hereditary spherocytosis, ${ }^{13}$ to trigger the development of jaundice in newborns.

Previous reports have provided strong arguments favouring the hypothesis that jaundice associated with hypertrophic pyloric stenosis is an early manifestation of Gilbert syndrome. In our two patients homozygous for the (TA) ${ }_{7}$ TAA polymorphism, the diagnosis of

This work was partially supported by a grant from the Délégation à la Recherche Clinique (CRC 950038). We thank Alan Strickland for editing the English.

1 Lippert MM. Pyloric stenosis presenting as severe prolonged jaundice. S Afr Med $\mathcal{F}$ 1986;69:446-7.

2 Alagille D, Odière M. Hyperbilirubinemia of the newborn. In: Alagille $\mathrm{D}$, Odièvre $\mathrm{M}$, eds. Liver and biliary tract diseases in children. New York: Wiley Medical, 1979:18-42.

3 Labrune P, Myara A, Huguet P, Trivin F, Odièvre $M$. Jaundice with hypertrophic pyloric stenosis: a possible early manifestation of Gilbert syndrome. F Pediatr 1989;115:93-5.

4 Bosma PJ, Roy Chowdhury J, Bakker G, et al. The genetic basis of the reduced expression of bilirubin UDPbasis of the reduced expression , of bilirubin UDPMed 1995;333:1171-5.

5 Monaghan G, Ryan M, Seddon R, Hume R, Burchell B. Genetic variation in bilirubin UDP-glucuronosyltransferase gene promoter and Gilbert's syndrome. Lancet 1996;347:578-81. 
6 Yamamoto K, Sato H, Fujiyama Y, Doida Y, Bamba T. Contribution of two missense mutations (G71R and Y486D) of the bilirubin UDP glycosyltransferase (UGT1A1) gene to phenotypes of Gilbert's syndrome and Crigler-Najiar synd

7 Maruo Y, Sato H, Yamamoto T, Doida Y, Shimada M. Gilbert syndrome caused by a homozygous missense mutation (Tyr486Asp) of bilirubin UDP-glucuronosyltransferase gene. F Pediatr 1998;132:1045-7.

8 Bosma PJ, Roy Chowdhury N, Goldhoorn BG, et al. Sequence of exons and the flanking regions of human bilirubin-UDP-glucuronosyltransferase gene complex and identification of a genetic mutation in a patient with Crigler-Najiar syndrome type I. Hepatology 1992;15: 941-7.

9 Beutler E, Gelbart T, Demina A. Racial variability in the UDP-glucuronosyltransferase 1 (UGT1A1) promoter: a balanced polymorphism for regulation of bilirubin metabolism. Proc Natl Acad Sci USA 1998;95:8170-4.
10 Bancroft JD, Kreamer B, Gourley GR. Gilbert syndrome accelerates development of neonatal jaundice. $\mathcal{F}$ Pediatr 1998;132:656-60.

11 Monaghan G, McLellan A, McGeehan A, et al. Gilbert's syndrome is a contributory factor in prolonged unconjugated hyperbilirubinemia of the newborn. If Pediatr 1999;134:441-6.

2 Kaplan M, Renbaum P, Levy-Lahad E, Hammerman C, Lahad A, Beutler E. Gilbert syndrome and glucose-6phosphate dehydrogenase deficiency: a dose-dependent genetic interaction crucial to neonatal hyperbilirubinemia. Proc Natl Acad Sci USA 1997;94:12128-32.

13 Iolascon A, Faienza MF, Moretti A, Perrotta S, Miraglia del Giudice E. UGT1 promoter polymorphism accounts for increased neonatal appearance of hereditary spherocytosis [letter]. Blood 1998;91:1093.

14 Clarke DJ, Moghrabi N, Monaghan G, et al. Genetic defects of the UDP-glucuronosyltransferase-1 (UGT1) gene that of the UDP-glucuronosyltransferase-1 (UGT1) gene that
cause familial non-haemolytic unconjugated hyperbilirubinaemias. Clin Chim Acta 1997;266:63-74. 